Article

\title{
Broadening of Light Coupling to Waveguide Modes in Solar Cells by Disordered Grating Textures
}

\author{
Karsten Bittkau ${ }^{1, *}(\mathbb{D})$, André Hoffmann ${ }^{1}$, Ulrich W. Paetzold ${ }^{1,2,3}$ and Reinhard Carius ${ }^{1}$ \\ 1 IEK5-Photovoltaik, Forschungszentrum Jülich GmbH, 52425 Jülich, Germany; \\ andre.hoffmann@rwth-aachen.de (A.H.); ulrich.paetzold@kit.edu (U.W.P.); r.carius@fz-juelich.de (R.C.) \\ 2 Institute for Microstructure Technology, Karlsruhe Institute of Technology (KIT), \\ Hermann-von-Helmholtz-Platz 1, 76344 Eggenstein-Leopoldshafen, Germany \\ 3 Light Technology Institute, Karlsruhe Institute of Technology (KIT), Engesserstraße 13, \\ 76131 Karlsruhe, Germany \\ * Correspondence: k.bittkau@fz-juelich.de; Tel.: +49-2461-6115-53
}

Academic Editor: Chien-Hung Liu

Received: 20 June 2017; Accepted: 13 July 2017; Published: 15 July 2017

\begin{abstract}
We investigated the light coupling to waveguide modes in nanophotonic thin-film solar cells exhibiting a tailored disordered grating texture by rigorous optical simulations. Such disordered nanophotonic light trapping concepts have demonstrated enhanced short-circuit current density compared to ordered nanophotonic thin-film solar cells. This observation is commonly explained by a spectral broadening of the resonant light coupling to waveguide modes. In this work, we investigated the origin of this spectral broadening. We identified two basic mechanisms that lead to a spectral broadening of the light coupling to waveguide modes: (1) the broadening of the wave vector transferred by the disordered interface texture and (2) the broadening of the waveguide mode due to the distortion of the wave guiding absorber layer. Depending on the type of disorder, the contribution from each of the mechanisms varies.
\end{abstract}

Keywords: Light trapping; thin-film solar cells; waveguide modes; optical simulation; FDTD; disordered gratings

\section{Introduction}

Advanced light-trapping concepts are one way to enhance light absorption in the absorber layer and thereby improve solar cell efficiencies. This holds in particular for thin absorber layers, where the thickness of the device gets similar to or smaller than the absorption length of impinging light in a broad spectral range. In such thin devices, the resonant coupling to waveguide modes is of utmost importance [1]. Since resonant light coupling to waveguide modes is only possible in small spectral regions compared to the broad solar spectrum, an engineered light-trapping design to address the whole spectrum efficiently is necessary.

Periodic light trapping textures have demonstrated strong enhancement of the external quantum efficiency (EQE) of solar cells in spectrally sharp resonances [2,3]. In contrast, solar cells with random textures show a spectrally broad improvement of EQE [4-7]. However, due to the missing optical resonance, this improvement remains below the values for solar cells with periodic textures at their resonances [8].

Disordered grating textures offer the opportunity to couple incident light to guided modes in the absorber layer within a spectrally broader regime with a slightly reduced maximum, as compared to the well-ordered grating textures. It was shown experimentally that such disordered grating textures can improve the overall efficiency of the solar cells even further compared to the well-ordered grating textures [9].

In this study, we investigate the impact of disordered grating textures on the EQE of solar cells by applying the Finite-Difference Time-Domain (FDTD) method [10,11]. We used a hydrogenated 
amorphous silicon (a-Si:H) thin-film solar cell in superstrate configuration with a nominal absorber layer thickness of $250 \mathrm{~nm}$ as a test device. The disorder is introduced by two different ways, a variation of the particle position within the unit cell while keeping the shape of the particles constant, and a variation of the radius of the particles while keeping the position of the particles within the unit cell constant. The simulations were performed in the relevant spectral range for light trapping with a spectral resolution of $2 \mathrm{~nm}$ in order to resolve the resonances accurately.

By taking into account the Fourier transform of the interface topography as well as the local layer thickness, we show that the improvement in EQE due to the disorder can be explained by both a spectral broadening of the waveguide mode itself and a diffuse wave vector transfer at the disordered grating texture. We show that the disorder in the particle position dominantly leads to a diffuse wave vector transfer, while the disorder in the particle radius dominantly leads to a broadening of the waveguide mode itself.

\section{Structure and Method}

\subsection{Device Structure}

In this work, hydrogenated amorphous silicon (a-Si:H) thin-film solar cells are simulated as test devices. The advantage of these devices is given by the soft band edge which results in a wide spectral region of weak absorptance. Here, any prolongation of the effective light path, in particular the excitation of waveguide modes, significantly improves the generated photocurrent. Furthermore, a-Si:H solar cells can be fabricated on a large variety of textured substrates with comparable material properties [9]. This is due to the amorphous growth, which results in a relaxed constraint between the absorber material and the substrate.

The investigated solar cell structure is the widely used superstrate configuration, where the light illuminates through a transparent superstrate, i.e. glass. On top of the glass, a transparent conductive oxide (TCO) - in our case an 80-nm thick aluminum-doped zinc oxide-is placed, which forms the front contact layer of the solar cell. Afterwards, a 250-nm thick a-Si:H slab is placed, representing the layer stack of p-doped, intrinsic, and n-doped a-Si:H of the solar cell. The layer stack is finalized by the back-reflecting contact consisting of an 80-nm thick TCO and a perfect electric conductor (PEC) half space. The nanophotonic light trapping texture is assumed at the interface between the glass superstrate and the front TCO layer. The textures are two-dimensional gratings consisting of nanopillars capped by half-spheres. A sketch of the device for a period $p$ of $500 \mathrm{~nm}$ is shown in Figure 1a.

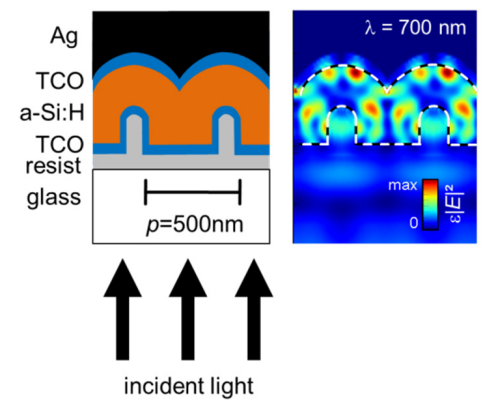

(a)

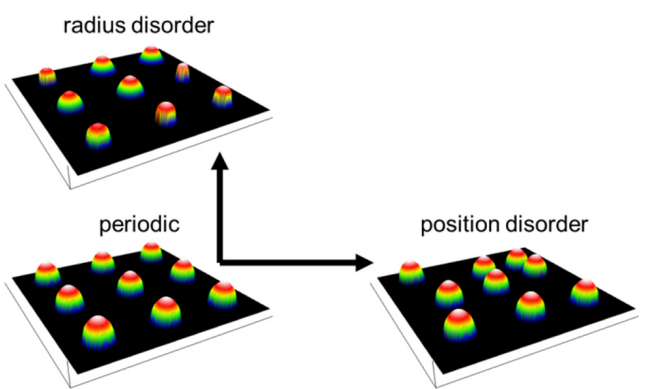

(b)

Figure 1. (a) Schematic illustration of the nanophotonic thin-film solar cell. The frontside is textured with a periodic array of nanopillars (gray) in the glass superstrate (white) capped by half-spheres and a transparent conductive oxide layer (TCO) as the front contact (blue). The a-Si:H layer (orange) forms the solar cell absorber which is finalized with a back reflector consisting of a TCO (blue) and a perfect electric conductor (black). A cross-section image of the simulated electric field energy for a wavelength of $700 \mathrm{~nm}$ is shown on the right side. (b) Illustration of different ways of disorder which are introduced to the grating structures. 


\subsection{Optical Simulations}

The correlation between light absorption, i.e. current generation of the solar cells and the various nanophotonic grating textures, is investigated by rigorous optical simulations. We used a home-built interface to the Finite-Difference Time-Domain solver Meep [11]. The experimentally determined optical data are taken as input for the simulations. An isotropic growth model [12] is assumed to take into account the changes in the interface texture due to the coverage of the texture by the a-Si:H layer. A monochromatic plane wave source is implemented in the incident glass half space. Periodic boundary conditions are assumed in the lateral direction and open boundaries in the propagation direction. The full three-dimensional electromagnetic field distribution at steady-state condition is the output of the simulation. From this output, the optical absorptance in each layer is extracted. We consider that each photon that is absorbed in the a-Si:H slab contributes to the generated photocurrent, whereas absorption in any other layer is considered to be parasitic. A spectral resolution of $2 \mathrm{~nm}$ is used in order to resolve the resonances of the waveguide modes. For the perfect periodic grating textures, one lateral unit cell is taken as the calculation domain. In the case of the disordered gratings, a lateral domain of $5 \times 5 \mu \mathrm{m}^{2}$ was chosen. The domain size in the propagation direction was $2 \mu \mathrm{m}$.

An example of the simulation results is shown in the right part of Figure 1a for an incident wavelength of $700 \mathrm{~nm}$. The interfaces of the absorber layer are depicted by the dashed lines. In the perfect periodic case, the excitation of a waveguide mode can be seen in Figure 1a by the well-defined intensity pattern in the high refractive index absorber medium. Such waveguide modes exhibit a resonant behavior with distinct sharp maxima in the absorptance. This resonance is achieved for the wavelength where the planar wave vector of the waveguide mode $\left(k_{\|}\right)$equals an integer multiple $n$ of the lattice wave vector:

$$
k_{\|}=n \cdot 2 \pi / g,
$$

where $g$ is the grating constant, i.e., the period.

Since the resonances related to waveguide modes are spectrally narrow, we introduce a tailored disorder to the grating structure in order to spectrally broaden the absorption enhancement. Two kinds of disorder are considered: position disorder and radius disorder. For both cases, the respective parameter is varied with respect to the perfect periodic structure according to a Gaussian distribution defined by the standard deviation $\sigma$. This leads to disordered grating textures with particles with identical shape but a random position within the unit cell, in the case of the position disorder. For the radius disorder, the particles are located at the same position within the unit cells but their shape is varied by a randomized radius. Examples of the assumed interface textures for the periodic case and for the position and radius disorder are illustrated in Figure $1 b$.

\section{Results and Discussion}

\subsection{Spectral Broadening of Light Coupling to Waveguide Modes}

Generally, the light coupling to waveguide modes in a photonic device with disordered gratings reveal a spectral broadening compared to the situation without disorder. This can be explained by (1) the broadening of the wave vector transfer and (2) the broadening of the waveguide modes. Both increase the total coupling of the incident light to waveguide modes. The former process leads to a certain range of wave vectors that is transferred to incident photons. Due to the dispersion relation of the waveguide mode, this range is translated to spectrally broader resonance energy. The latter process results in an internal optical damping of the waveguide mode, which is directly accompanied by broader resonance. This means that both possible processes lead to a broadening of the resonances in an optical experiment. Therefore, it is difficult to derive their contribution directly.

In the following sections, we demonstrate that the dominating process of spectral broadening of light coupling to waveguide modes depends on the way in which disorder is introduced to the nanophotonic grating structure. For this work, a texture analysis based on fast Fourier transformation 
(FFT) is applied as well as an analysis of the local absorber layer thickness variation. Finally, the impact of the disordered grating textures on the spectral response of the solar cells is investigated.

\subsection{Texture Analysis}

In order to prove a possible broadening of the transferred wave vector, we investigated the variety of applied disordered interface textures. A perfect periodic interface structure reveals a well-defined wave vector transfer by light diffraction into different orders. The transferred wave vector can be directly determined from the period of the grating. In the case of a disordered texture, it is necessary to apply a Fourier analysis to obtain the distribution of lateral feature sizes. Perfect periodic gratings show distinct peaks in the Fourier transform, whereas fully randomized textures result in a broad Fourier spectrum. In other words, any possible broadening of the transferred wave vector in a disordered grating texture is connected to a broader peak in the Fourier transform of the texture. Therefore, FFT is applied to our assumed disordered grating textures in order to evaluate the broadening of the transferred wave vector.

Figure 2 shows selected interface topographies and their corresponding FFT images for the case of the perfect periodic grating, the disordered texture assuming a position disorder with $\sigma_{p}=22 \mathrm{~nm}$, and the disordered texture with an introduced radius disorder of $\sigma_{r}=32 \mathrm{~nm}$. As expected, the FFT image of the perfect periodic grating structure only consists of distinct peaks at integer multiples of the reciprocal grating period. This reflects the well-defined wave vector transfer when a periodic texture is applied to a wave guiding slab. For both disordered textures, a diffusive signature is visible in the FFT images. For the structure with radius disorder (Figure 2c), there is only a weak diffusive background visible in the FFT (Figure 2f) where the peaks related to the diffraction orders are still dominating. In contrast, the diffusive part strongly dominates the FFT in the case of an applied position disorder (Figure 2e). Note that the applied standard deviations cannot be directly compared as their boundaries are different. It is therefore concluded that position disorder influences the wave vector transfer much more strongly than radius disorder.

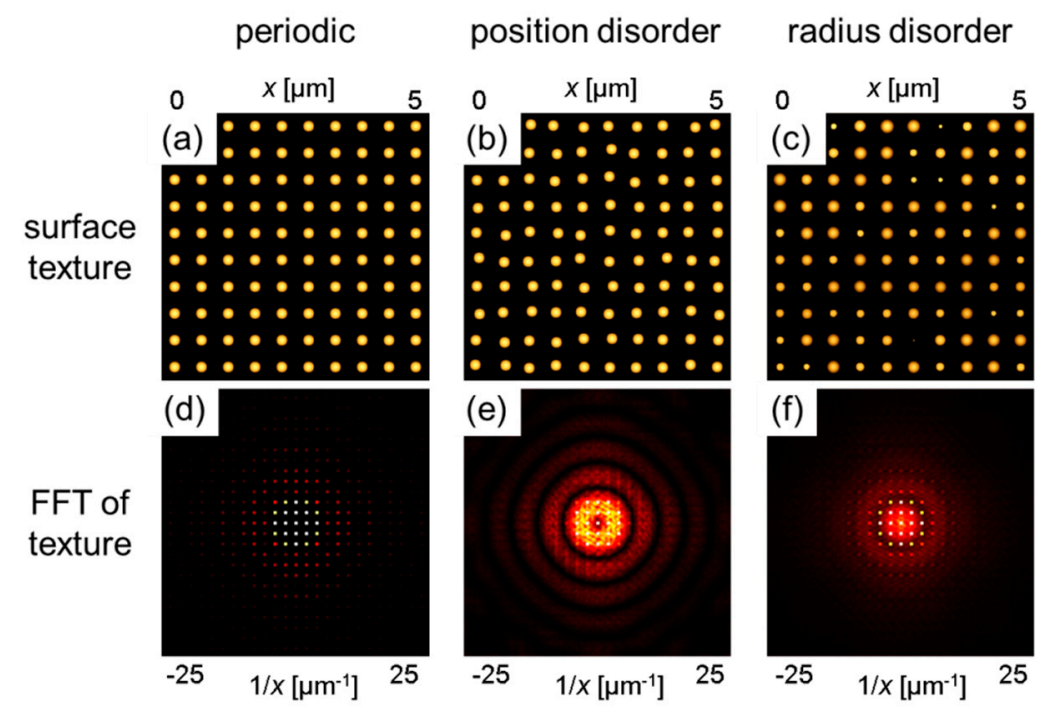

Figure 2. (a-c) Representative topography images of the periodic grating texture, the texture with introduced position disorder and with radius disorder, respectively. (d-f) The corresponding images of the 2D-Fast Fourier transformation (FFT) applied to the textures shown in (a-c), respectively.

\subsection{Local Layer Thickness}

It is difficult to quantify the impact of the interface structure of a wave guiding slab on the spectral width of the light coupling to the resonance mode as, without any interface structure, this 
light coupling is not possible at all. The situation becomes much more complex when looking for the impact of disorder within a grating coupler on the spectral width of the waveguide mode. On the one hand, the waveguide mode is already influenced in the case of the ordered grating structure. On the other hand, the disorder in the grating structure already influences the wave vector that is transferred to incident photons in a way that the resonance mode spectrally broadens.

In the present study, the wave guiding slab is grown on top of a textured substrate, which means that the interfaces of the slab are corrugated. In particular, the growth of a-Si:H can be assumed to be isotropic, which leads to a modification of the backside texture as compared to the frontside texture [12]. Due to this isotropic growth, the thickness of the wave guiding layer fluctuates along the surface texture when the thickness is defined along the intentional growth direction. Since its thickness is a main parameter to determine the resonance condition of a wave guiding slab, the disorder in the local layer thickness behavior can be used to investigate the impact of the disorder on the quality of the wave guiding properties.

Figure 3 shows the height profiles for the frontside texture (black lines), the backside texture (red lines) and the corresponding local layer thickness (red dashed lines), which is defined by the difference between the height profile at the backside and at the frontside. The results for the perfect periodic case are shown in Figure 3a, while those for a representation of a radius disorder introduced into the grating structure are shown in Figure $3 \mathrm{~b}$. Although the frontside textures of both structures (periodic and with radius disorder) look quite different from each other, the corresponding backside textures appear to be very similar. Small differences can only be obtained by a close look. This is a result of the isotropic growth of a-Si:H. As a consequence, the local layer thicknesses show significant differences depending on the applied disorder.

In order to visualize this for the different investigated textures, top view images of the frontside texture, the backside texture, and the local layer thickness are illustrated in Figure 4 for the perfect periodic case and the structures with position disorder and radius disorder. As expected, the frontside textures (Figure $4 \mathrm{a}-\mathrm{c}$ ) reveal significant differences for the differently applied disorder. The backside textures (Figure $4 \mathrm{~d}-\mathrm{f}$ )) are more or less equalized for the perfect periodic case and the texture with radius disorder. Significant differences were only found for the texture with position disorder. This is a result of the isotropic growth of a-Si:H on top of the structured interface. Due to this growth behavior, the radius of the half-conically shaped nanostructures increases with the layer thickness. As long as the layer thickness reaches or exceeds half the distance between the nanostructures, the resulting radius is defined by the layer thickness rather than the original radius.

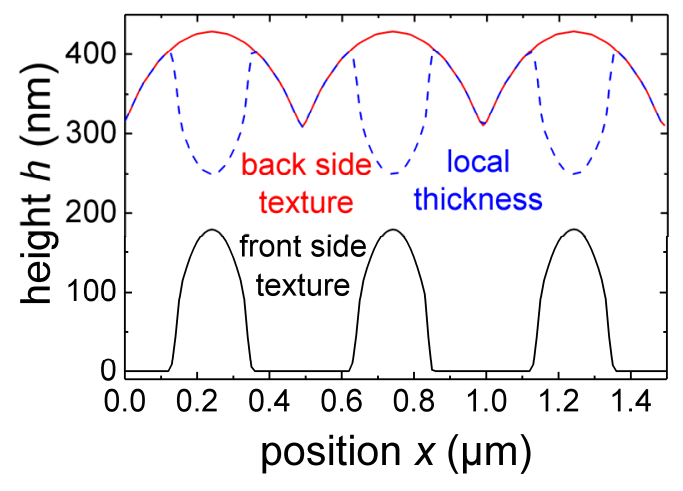

(a)

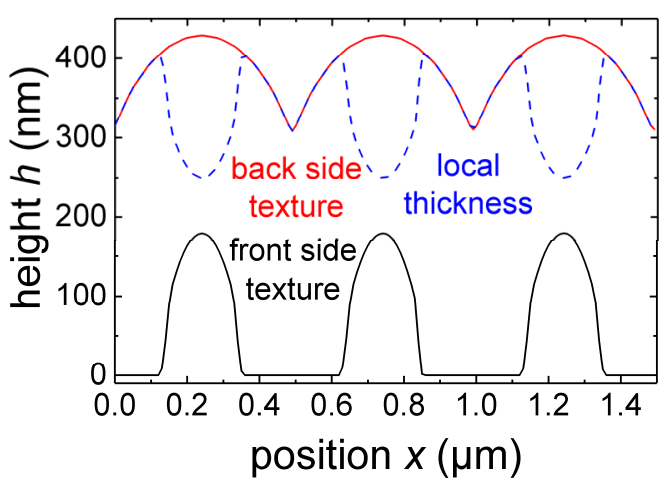

(b)

Figure 3. (a) Height profile of the frontside (black) and backside (red) texture for the perfect periodic texture. The dashed blue line indicates the local thickness of the wave guiding slab along the line profile. (b) The same quantities are shown for a special representation of an introduced radius disorder into the grating structure. 
This has a strong impact on the local layer thickness, as can be seen in Figure 4g-i. Each unit cell shows a typical characteristic in the perfect periodic case, which is quite similar to the shape of the unit cells for the texture with position disorder applied to the grating. The waveguide resonance can be understood as laterally coupled optical resonators with each unit cell forming a resonator. When each resonator exhibits the same resonance energy, the lateral interconnection leads to an extended optical mode, the waveguide mode. In the case of the texture with radius disorder, the shape of the different resonators differs significantly from each other. Therefore, the formation of an extended optical mode is hindered. In other words, any guided mode is damped, which leads to a spectrally broader resonance.

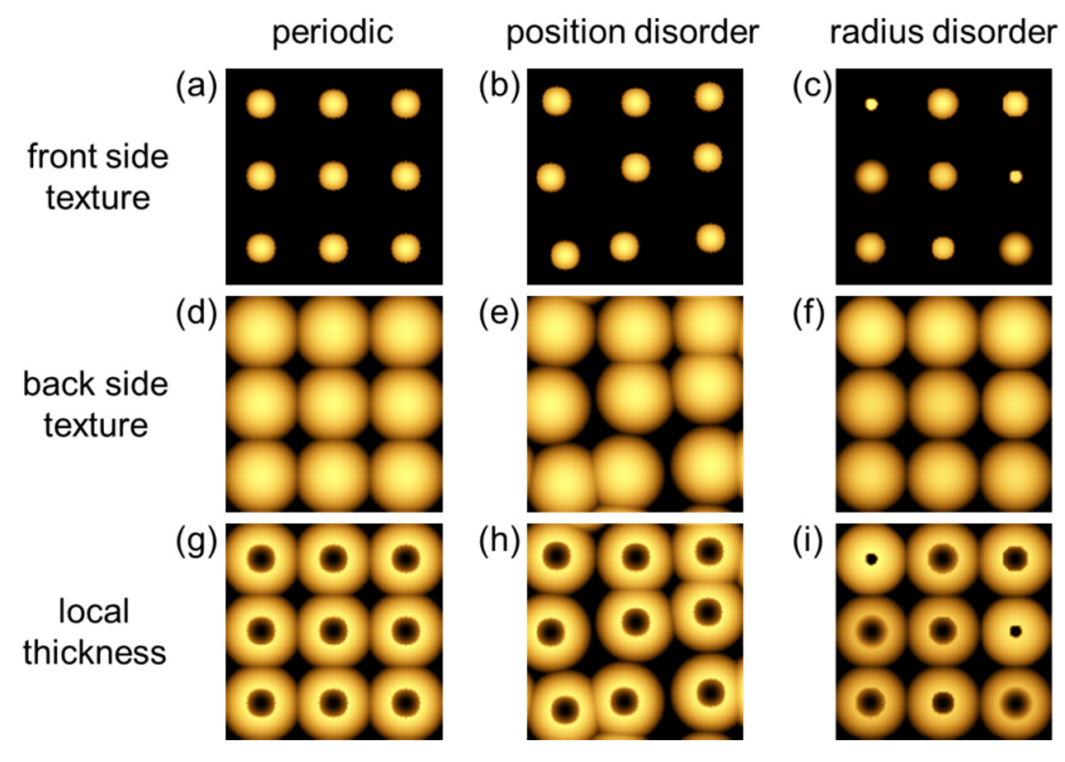

Figure 4. Top view images of the frontside texture $(\mathbf{a}-\mathbf{c})$, backside texture $(\mathbf{d}-\mathbf{f})$, and local layer thickness (g-i) for the perfect periodic grating and the gratings with position and radius disorders.

From this part of the investigation, it is concluded that radius disorder has a much stronger influence on the spectral width of the waveguide mode than position disorder. It should be kept in mind that, in contrast, the position disorder has a stronger impact on the wave vector transfer than radius disorder. With these two ways of implementing disorder in a grating structure, the investigation of the impact of these two origins of broadening on the performance of a spectrally broad optoelectronic device such as a thin-film solar cell is now possible.

\subsection{Impact of Disorder in Solar Cells}

In order to evaluate the impact of the disordered grating textures on the broadband performance of the a-Si:H solar cell, we simulated the EQE in the spectral range from $635 \mathrm{~nm}$ to $780 \mathrm{~nm}$ with a spectral resolution of $2 \mathrm{~nm}$. For shorter wavelengths, the absorption coefficient of a-Si:H is too high for the formation of waveguide modes; for longer wavelengths, the absorption coefficient is too low to contribute significantly to the photocurrent generation at the given cell thickness. The results are shown in Figure 5. Figure 5a,b illustrate the broadening of the spectral resonances in the EQE due to the position disorder and radius disorder, respectively. In both illustrations, the black line corresponds to the perfect periodic case, which is characterized by distinct maxima in the EQE which are related to the resonant light coupling of incident light to waveguide modes in the absorber layer of the solar cell. The EQEs for the implementation of a moderate disorder into the grating textures are shown as red lines. These curves still show some resonant behavior, but with strongly reduced peak heights which are spectrally much broader. The blue curves depict the situation for a high degree of disorder. Resonant behaviors are no longer visible and the shape of the curves is smooth. 
Obviously both physical effects, the less well-defined wave vector transfer in the case of the position disorder as well as the less well-defined waveguide mode in the case of the radius disorder lead to the similar effect of a smearing out of the resonant behavior of light coupling of incident light to guided modes in the absorber layer of the solar cell. Nevertheless, the two ways of applying disorder into the grating textures show significant differences. In the case of the position disorder (Figure 5a), the EQE is reduced at the spectral positions of the waveguide modes (e.g., at $\lambda \sim 720 \mathrm{~nm}$ ) but reveal an overall improved EQE off of these spectral resonance positions (e.g., at $700 \mathrm{~nm}<\lambda<715 \mathrm{~nm}$ or $725 \mathrm{~nm}<\lambda<750 \mathrm{~nm}$ ). This already suggests an overall improvement of photocurrent generation due to the implementation of position disorder into the grating texture. In contrast, the EQE curves in the case of the grating textures with radius disorder show spectral regions where the EQE is improved, but also other regions with a reduced response (e.g., at $670 \mathrm{~nm}<\lambda<690 \mathrm{~nm}$ ). In order to quantify the impact of the different ways of disorder on the solar cell performance in the broad spectral range, the photocurrent is determined for each structure and the results are compared to each other.

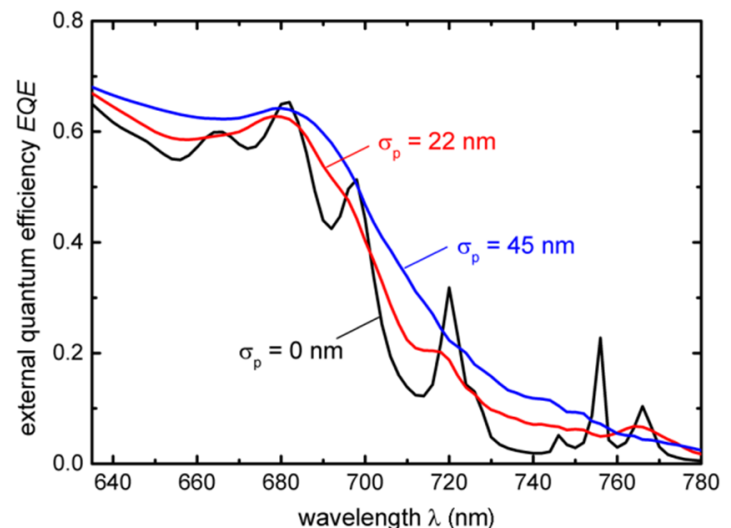

(a)

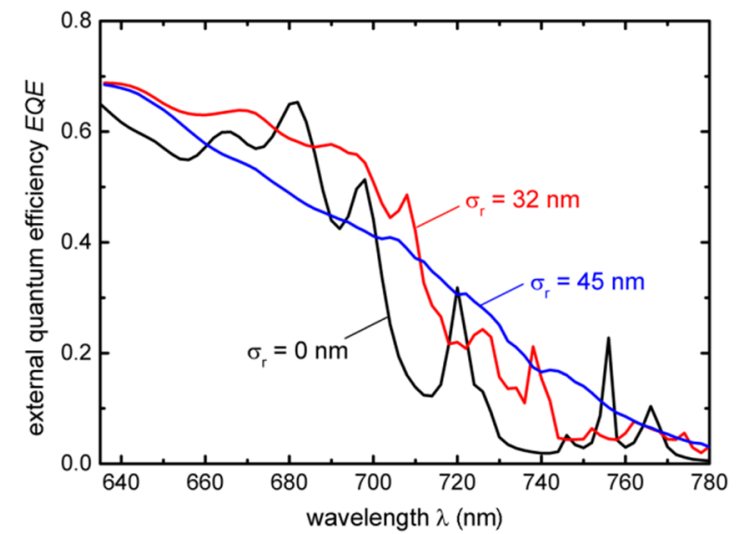

(b)

Figure 5. (a) External quantum efficiency of a-Si:H solar cells with different degrees of position disorder in the grating textures. The position disorder is parameterized by the Gaussian width $\sigma_{\mathrm{p}}$. (b) External quantum efficiency of a-Si:H solar cells with different degrees of radius disorder in the grating textures. The radius disorder is parameterized by the Gaussian width $\sigma_{\mathrm{r}}$.

Therefore, the simulated EQE curves are weighted by the solar irradiance and spectrally integrated to obtain the short-circuit current density (JSC) of the solar cells generated in the spectral region from $635 \mathrm{~nm}$ to $780 \mathrm{~nm}$. The results are plotted in Figure 6 as a function of the respective disorder parameter. We found that an increase of position disorder monotonically enhances the short-circuit current density (blue solid line). At larger disorder, JSC of the solar cell saturates. The radius disorder (red dashed line) shows a different trend. For smaller disorder parameters up to $45 \mathrm{~nm}$, the JSC increases. Afterwards, the short-circuit current density decreases while still surpassing the level of the well-ordered periodic texture.

Both investigated ways of introducing disorder into the grating structure lead to an improvement of $\mathrm{J}_{\mathrm{SC}}$ of the solar cell as long as the strength of disorder is moderate. With a higher degree of disorder, the impact on the solar cell performance varies for the different ways of disorder. The JSC of the solar cells with position disorder in the grating texture saturates. In contrast, solar cells with a higher degree of radius disorder exhibit lower JSC than solar cells with a moderate degree of radius disorder. This different behavior is explained as follows.

In the case of the position disorder, the improvement of JSC is mainly explained by a diffuse wave vector transfer. This follows from the Fourier transforms of the topographies of the different disordered grating textures, which show the strongest diffuse part in the case of the position disorder. 
The increase of $\mathrm{J}_{\mathrm{SC}}$ in the case of the radius disorder is mainly explained by a broadening of the waveguide mode. Here, the Fourier transforms of the textures show only a weak diffuse part. Furthermore, the local layer thickness varies strongly from unit cell to unit cell due to the isotropic growth of the a-Si:H layer. This leads to a broadening of the resonance condition of the waveguide mode. A further increase of the radius disorder results in a disturbance of the waveguide mode because the differences between the individual resonances of the unit cells become too large to form an extended optical mode in the layer. This explains the decrease of JSC for larger radius disorder.

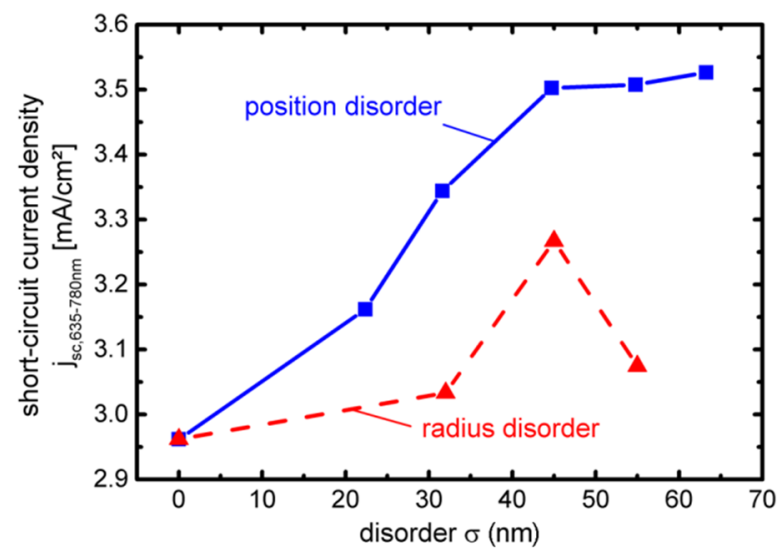

Figure 6. Impact of different types of disorder on the short-circuit current density of the a-Si:H solar cell in the wavelength range from $635 \mathrm{~nm}$ to $780 \mathrm{~nm}$.

The two presented ways of implementing disorder into a grating texture are merely two possible examples. Of course, there are many other possibilities to vary the shape of the particles or their spatial distribution. The investigated position and radius disorder represent two extreme cases where the two basic physical mechanisms are dominating, respectively. How other types of disorder impact the light coupling to waveguide modes and, thereby, solar cell efficiency is open to further research.

\section{Conclusions}

We investigated the physical mechanism of the spectrally broad light coupling to waveguide modes in thin-film solar cells by disordered grating textures at the interfaces of the absorber layer by rigorous optical simulations with FDTD. We demonstrated that the improvement of the short-circuit current density of the solar cells achieved by introducing disorder to the grating, compared to the ordered grating, is due to a combination of a broadening of the wave vector transfer by the disordered grating texture and a spectral broadening of the waveguide mode itself. Our conclusions are supported by a detailed FFT analysis of the interface textures and an analysis of the variation of the shape of the unit cells, taking into account the isotropic growth of the absorber layer. We found that the broadening of the wave vector transfer by the disordered grating texture leads to an improvement of short-circuit current density with a saturation behavior. The broadening of the waveguide mode itself leads to an improvement of short-circuit current density only for moderate disorder due to the disturbance of the extended optical mode. Therefore, the improvement of short-circuit current density is reduced for higher disorder in this case.

Acknowledgments: The authors thank Dmitry Chigrin, Gero von Plessen and Thomas Taubner from RWTH Aachen for fruitful discussions. The FDTD simulations are carried out at the JURECA HPC cluster at Jülich Supercomputing Center in the frame of the VSR project JIPV00. The financial support of the Helmholtz Association's Initiative and Networking Fund for the Helmholtz Young Investigator Group of Ulrich Paetzold is highly acknowledged.

Author Contributions: Karsten Bittkau wrote the paper, André Hoffmann performed the optical simulations. Karsten Bittkau and André Hoffmann designed the input structures and analyzed the data. Ulrich W. Wilhelm 
Paetzold and Reinhard Carius supported the work with discussions and helped with the interpretations of the results.

Conflicts of Interest: The authors declare no conflict of interest.

\section{References}

1. Yu, Z.; Raman, A.; Fan, S. Fundamental limit of nanophotonic light trapping in solar cells. Proc. Natl. Acad. Sci. USA 2010, 107, 17491-17496. [CrossRef] [PubMed]

2. Ferry, V.E.; Verschuuren, M.A.; van Lare, M.C.; Schropp, R.E.I.; Atwater, H.A.; Polman, A. Optimized Spatial Correlations for Broadband Light Trapping Nanopatterns in High Efficiency Ultrathin Film a-Si:H Solar Cells. Nano Lett. 2011, 11, 4239-4245. [CrossRef] [PubMed]

3. Paetzold, U.W.; Moulin, E.; Michaelis, D.; Böttler, W.; Wächter, C.; Hagemann, V.; Meier, M.; Carius, R.; Rau, U. Plasmonic reflection grating back contacts for microcrystalline silicon solar cells. Appl. Phys. Lett. 2011, 99, 181105. [CrossRef]

4. Tiedje, T.; Yablonovitch, E.; Cody, G.D.; Brooks, B.G. Limiting efficiency of silicon solar cells. IEEE Trans. Electron Devices 1984, 31, 711-716. [CrossRef]

5. Kluth, O.; Rech, B.; Houben, L.; Wieder, S.; Schöpe, G.; Beneking, C.; Wagner, H.; Löffl, A.; Schock, H.W. Texture etched $\mathrm{ZnO}: \mathrm{Al}$ coated glass substrates for silicon based thin film solar cells. Thin Solid Films 1999, 351, 247-253. [CrossRef]

6. Krc, J.; Zeman, M.; Kluth, O.; Smole, F.; Topic, M. Effect of surface roughness of ZnO:Al films on light scattering in hydrogenated amorphous silicon solar cells. Thin Solid Films 2003, 426, 296-304. [CrossRef]

7. Fay, S.; Steinhauser, J.; Oliveira, N.; Vallat-Sauvain, E.; Ballif, C. Opto-electronic properties of rough LP-CVD ZnO:B for use as TCO in thin-film silicon solar cells. Thin Solid Films 2007, 515, 8558-8561. [CrossRef]

8. Battaglia, C.; Hsu, C.-M.; Söderström, K.; Escarré, J.; Haug, F.-J.; Charrière, M.; Boccard, M.; Despeisse, M.; Alexander, D.T.L.; Cantoni, M.; Cui, Y.; Ballif, C. Light Trapping in Solar Cells: Can Periodic Beat Random? ACS Nano 2012, 6, 2790-2797. [CrossRef] [PubMed]

9. Paetzold, U.W.; Smeets, M.; Meier, M.; Bittkau, K.; Merdzhanova, T.; Smirnov, V.; Michaelis, D.; Wächter, C.; Carius, R.; Rau, U. Disorder improves nanophotonic light trapping in thin-film solar cells. Appl. Phys. Lett. 2014, 104, 131102. [CrossRef]

10. Taflove, A.; Hagness, S.C. Computational Electrodynamics: The Finite-Difference Time-Domain Method, 3rd ed.; Artech: Norwood, MA, USA, 2000.

11. Oskooi, A.F.; Roundy, D.; Ibanescu, M.; Bermel, P.; Joannopoulos, J.D.; Johnson, S.G. MEEP: A flexible Free-software package for electromagnetic simulations by the FDTD method. Comput. Phys. Commun. 2010, 181, 687-702. [CrossRef]

12. Jovanov, V.; Xu, X.; Shrestha, S.; Schulte, M.; Hüpkes, J.; Zeman, M.; Knipp, D. Influence of interface morphologies on amorphous silicon thin film solar cells prepared on randomly textured substrates. Sol. Energy Mater. Sol. Cells 2013, 112, 182-189. [CrossRef] 